\title{
Editorial: Why a European Journal of Workplace Innovation?
}

Øyvind Pålshaugen 


\section{Introduction}

The expansion of the 'field of innovation' that took place during the last quarter of the last century has in this century become almost overshadowed by the 'field of innovation studies'. What, then, is the rationale for launching EJWI in this new field? The name informs you that this new journal will address issues of innovation. In particular, it will address the kind of issues that arise from the simple and fundamental fact that innovation takes place at workplaces. This opening article will present the editors' perspectives on why there is a need for a journal, and on how to meet this need.

The field of innovation studies may rightly be called a 'new scientific field' (Fagerberg and Verspagen 2009). This field has not emerged in the form of a new scientific discipline. Rather, the field of innovation studies is characterised by multi- and cross-disciplinary approaches. Innovation studies are largely undertaken by research centres and research programmes that are not based on the traditional academic division of labour among scientific disciplines. In this way, the field of innovation studies shares some of the characteristics of the more established field of organisation studies, and e.g. feminist studies or cultural studies. In this way, "the development of innovation studies as a scientific field is part of a broader trend (...) that blurs traditional boundaries and challenges existing patterns of organisation within science (including social science)" (Fagerberg and Verspagen 2009: 218).

This broader trend, which challenges traditional forms of social science, also includes the increasing demand on social research to have some practical impact on its field of study. The rapid growth of the field of innovation studies throughout the last decades is part of, and partly caused by, the increased emphasis on innovation policy in Western countries. This emphasis has in turn been reinforced by theoretical and empirical results from innovation studies. In this way, there is a kind of 'social contract' between innovation policy and innovation studies at an overall societal level. In return for the investment in innovation studies, research that contributes to improving the conditions for, and practice of, innovation is expected.

This expectation means an increased demand on the social sciences engaged in the field of innovation studies. These are expected to contribute not only to developing new knowledge on innovation, but also to new practices of innovation. This demand has to be met in different ways, at different levels of innovation, and by different forms of innovation practice. Thus, it can be met by many kinds of research approaches.

However, no approach will be capable of meeting the demand of practical relevance in a way that will be useful at all levels of innovation policy, or to all forms of innovation practice. There is an obvious need for complementary approaches in the field of innovation studies. In this sense, this field is in need of pluralism, regarding both the approaches of research and the research policy to support these. EJWI aims to promote such pluralism. This plea for pluralism, however, is not a request for something like an "anything goes" attitude. What we need is rather a combination of an open and a critical attitude. To specify what that might mean, we have to take a brief, critical look at some general aspects of research practices in the field of innovation studies. 


\section{How innovation studies contribute to theoretical and practical results from research}

Common to all approaches that want to make themselves acknowledged as research approaches relevant in the field of innovation studies, is that they have to legitimise their relevance, in terms of their ability both to produce general scientific knowledge, and to produce knowledge that somehow is useful in practice. These are pretty strong demands, especially when taken together, as is the case in the field of innovation studies, at least in principle.

Some find that ideally, these requirements should be met by means of the one and same process of knowledge generation. There are two quite different types of research approach that claim to be able to realise this ideal. These two types may be placed in each end of a common axis. At the one end you will find the most 'purely theoretical' approaches, which claim that scientific knowledge from social research is inherently actionable, insofar it has general validity. At the other end, you will find the type of approaches that are based on some kind of practical cooperation with actors in the field (action research), and which claim that interactive relations between researchers and actors in the field are a condition for the creation of knowledge that is both actionable and valid.

Between these two poles, there is a wide range of approaches that do not claim to be able to deal with the request for both theoretical and practical results by one and the same methodology. Most of the research approaches within the field of innovation studies have a more pragmatic attitude to this demand. The most common way to deal with it is to consider it primarily as a question of mediation. From this pragmatic perspective, the purpose of the research process is to produce new knowledge, and the practical impact of this new knowledge is to be obtained by processes of mediation. Accordingly, this is the conventional division of labour within most research milieus. It is conventional wisdom that the task of mediating research results is a task to be dealt with after the research process is completed, and not as a part of it.

It has to be added that this conventional strategy of mediation has been rather successful within the field of innovation studies, considering the impact that results from social research on innovation seem to have had on the development of innovation policy throughout recent decades. The development of innovation policy, from focussing on research-based, radical innovation to a broader perspective, focussing on innovation as something that takes place within larger social systems, based on a number of factors and on experience-based as well as research-based forms of knowledge, is partly a result of the impact of (the field of) innovation studies. This impact is not to be regarded simply as a result of the scientific knowledge produced in this field; it is indeed a result also of the mediation of this knowledge: to a large extent carried out by innovation researchers, in a number of channels, arenas and forums.

Thus, the question of impact is most commonly considered, not as a task of research, but as an additional task for (some) researchers. Accordingly, the ambition of incorporating the demand for both theoretical and practical results into the research methodology is not very common in the field of innovation studies. However, the more extreme types of approaches mentioned above (which have this ambition), and the more common approaches to innovation studies, nevertheless have something in common. They all tend to consider research methodology as something that warrants the validity, or the scientific quality, of the research results. What differs is the extent to which they consider the demand for practical results of research projects to be relevant for questions of research methodology, and in what way. 


\section{How do innovation studies legitimate their scientific quality and practical relevance?}

This 'tacit consensus', among approaches that in many other respects are quite different from each other, and which are not just complementary but competing and even conflictual approaches, may appear pretty obvious. Both to the common researcher, as well as to the 'common man', it appears almost self-evident that the use of scientific methods is what makes knowledge generated by research fulfil the requirements for being considered scientific knowledge. However, from within the scientific community we also know very well that a minimum requirement is for a research project to fulfil the requirements for being able to producing scientific knowledge. What counts, both when judging an application for project funding, and in particular when judging the outcome of a project, is the 'scientific quality'.

The scientific quality may be judged 'low' or 'high', or anything between. When a social research project succeeds in producing results in the form of publications that are judged to have high scientific quality, this success is not just a function of the research methodology applied in the project. The intellectual and creative work of generating new insights and knowledge through the processes of analysing and reflecting upon the material, and the processes of writing a comprehensive scientific presentation, are the kinds of effort that usually make the difference between mediocre scientific works and works of higher scientific quality. We also know that different approaches of social research may differ quite a lot regarding to what extent they allow for such intellectual and creative efforts, in the process of generating new scientific knowledge.

Against this background, the 'tacit consensus' among the various research approaches within the field of innovation studies, on the need for any approach to have a coherent research methodology, appears to be a 'strategic consensus' rather than a real agreement. The main reason for this we probably find in the above-mentioned demand for both theoretical and practical results from innovation studies. Research approaches that are clever in contributing to practical results, but not in generating new knowledge, are to be aligned with consultancy agencies; and approaches that are clever in generating new knowledge, but with no practical impact, appear to be only of 'academic interest'.

Therefore, a challenge common to all kinds of approaches that want to be acknowledged as worthy of public (or private) funding within the field of innovation studies, is that they cannot legitimise their relevance by pointing at results, either in the form of theoretical knowledge or in the form of practical impact. Any approach has to legitimise its relevance along both theoretical and practical dimensions.

To cope with this 'double set' of demands to research approaches, the most common strategy has become to legitimise the capability of generating scientific knowledge by advocating a coherent research methodology. The strategies for legitimating that the scientific knowledge also is useful knowledge are more varied, but as we have seen, the most common is some strategy for mediation of scientific knowledge.

Whether the most common strategy of mediation is also the best one may be questioned, but we will leave this question here. Our concern in this editorial regards the common strategy of legitimising the scientific quality of one's research approach in its research methodology: that is, its theoretical framework and the associated methods of collecting and analysing data. This strategy works well vis á vis the research funding institutions, but does it work that well within the research community? 


\section{Consequences of using research methodology as the legitimacy base of approaches}

One unintended consequence of this strategy is that it reinforces some already prevalent tendencies within the communities of social research, and the scientific community in general. Firstly, it reinforces the well-known tendency of any particular approach of social research to defend its own research methodology, when questioned or challenged by other approaches. Secondly, it reinforces the tendency that most development of research approaches takes place inwards, so to speak, by developing new theoretical and methodical elements that aim at both expanding the approach and making it more comprehensive (not to say 'hegemonic'). Finally yet importantly, it strengthens the tendency to lack communication, and co-operation, across approaches of different kinds.

All these tendencies work together to form specific kinds of path-dependencies within each particular approach. The field of innovation studies as a whole suffers from these co-existing approaches, that create paths in the field which seldom cross other paths and allow for conversations at the cross-roads. Under these circumstances, the conditions for improving and increasing the theoretical and practical impact from innovation studies in the field of innovation are far from optimal.

Thus, there is a need for pluralism in the field of innovation studies, not only with regard to allowing for and stimulating the co-existence of different approaches. There is also a need for a kind of pluralism within each approach, and within the research projects undertaken by/from various approaches. The development and use of more flexible research methodologies, that is, a greater multitude of theoretical perspectives and a greater multitude of methods, within (and across) all approaches would certainly enhance the conditions for designing research projects whose outcome might have both higher scientific quality and higher relevance/practical impact. This thesis is a main element in the rationale of launching EJWI, and we will elaborate it a bit more.

\section{On understanding the larger totality and the need for a larger conversation}

As already stated, EJWI will emphasise the focus on 'workplace issues' in innovation research. Among the reasons for this is that we find that in the discourse on innovation policy, and in innovation studies today, many workplace-related aspects of relevance to understanding and improving the conditions for, and processes of, innovation are somewhat neglected. For example, many scientific publications based on some kind of 'innovation system' approach still tend to display rather 'distant' perspectives on the importance of the dynamic and the events at the workplace level, regarding theoretical, empirical and practical issues.

This of course does not mean that workplace-oriented studies of innovation should mean a narrow approach, expected to focus solely on the multitude of empirical factors and events that takes place within workplaces. A systemic approach is indeed required also when studying workplace issues related to innovation. However, a systemic approach to innovation studies is not to be equated with, or confused with, an innovation system approach (be it NIS or RIS).

Rightly, innovation system approaches usually represent some kind of comprehensive approaches to innovation studies. Nevertheless, the 'empirical' studies undertaken by any of these approaches, of course, do not comprehensively include an overall knowledge of all factors and aspects that may be of relevance for generating an adequate understanding of the innovation system which is the subject of study. 
In general, there is always the risk that innovation system approaches, because they are founded on substantial perspectives on what are the general features of the system under study, may miss opportunities to glimpse other substantial aspects than those that in principle are comprehended by the general perspectives that makes up the theoretical framework of an approach. Some of these aspects might be of crucial importance to an adequate understanding of what really takes place in the actual innovation system, but an innovation system approach may nevertheless systematically exclude them. This is why it is important to remember that systemic approaches in innovation studies are not to be identical to, or limited to, innovation system approaches.

This means that when we presume that all kinds of innovation somehow take place at workplaces, we by the same token presume that any workplace is part of a larger totality. Therefore, it is not possible to generate scientific knowledge on innovation that takes place at workplaces without taking this larger totality into consideration. However, we do not presume that any systemic approach to innovation studies can claim to offer a total overview.

What kind of systemic approach would be most apt in each particular study is thus dependent on the kind of issues of innovation we are studying, and on what may be adequate ways of conceptualising the larger totality of which these issues is a part. There is a mutual dependence between what aspects of innovation are to be focussed at the workplace, and what aspects of the larger totality are necessary to consider and understand. Therefore, there are no necessary preconceived conceptions of the larger totality that would be adequate to any particular research project.

Thus, a preconceived system theory of the larger totality within which innovations take place will not necessarily work as a general framework for generating scientific knowledge of all relevant aspects of this totality. This is why there is a need for a more flexible research methodology. In other words, there is a need for a pluralistic attitude, which allows us to draw on those theoretical perspectives and practical methods that are required by the kind of issues that are studied: regardless of whether the perspectives and methods are building blocks in, or part of, any particular approach. In this way, the generation of a scientific understanding of the larger totality that e.g. innovation at the workplace level unfolds within, requires participation in a larger scientific conversation among different approaches to innovation studies.

\section{And then, why EJWI?}

Against the background sketched above, EJWI aims to publish articles that contribute to the creation of knowledge on workplace-related issues of innovation that unfold within some kind of totality, a totality that no research approach can claim to fully overview. For these reasons, EJWI will advocate pluralism within innovation studies: a pluralism that is not just a question of the scientific attitude towards other approaches, but also a question of a critical attitude to one's own approach.

As will be understood by our readers, and by our writers, advocacy of this kind of pluralism will not mean an uncritical 'anything goes' publishing policy. For sure, we have no predetermined views on what content and form of articles will be most suitable to match, or outbid, the aims of EJWI in this respect. EJWI is literally and metaphorically open for any new contribution. However, we will indeed take on the efforts necessary to make critical judgements of whether the contributions written and submitted to be published in EJWI are also a contribution to the larger conversation among researchers from different approaches to innovation studies. 
This conversation, and the contributions to it, may deal with any issue, ranging from empirical details, via system models, to critical theory of science. However, what might unite the diverse kinds of contributions, and justify their publication, would be that they aim at a better understanding of any subject of innovation that in fact has to be studied as part of a larger totality in order to be adequately understood. This is regardless of, or rather because of, the fact that no approach and no study will comprise a full understanding of the larger totality that is invoked as the context of any particular text that is published. Nevertheless, any text may profit from being part of this larger conversation.

\section{And how?}

The ambition of EJWI to contribute to this larger conversation is not based on some simplified notions of what it means, and what it requires to realise it in our practice of publishing. Rather the opposite: this ambition is rooted in long-term experiences with the difficulties of making such conversations happen. For the very same reasons, the ambition is based on the acknowledgement of the need to improve the conditions for such conversations within the scientific community, and in its interfaces with the public sphere.

Today, when the abundance of scientific journals is so large that it is no longer the subscription to any particular journal, but the purchase of 'packets' of innumerable number of electronic journals which has become the standard practice of scientific institutions, it may appear somewhat untimely to launch a new journal that is based on the ambitions sketched above. As a means of scientific conversation, journals are only exceptionally approached or looked into because of the editor's intention or ambition with the journal. Searching articles by means of keywords, regardless of in which particular journal the searched article is published, has become the current mode of seeking 'conversation partners'. Moreover, articles are often searched not for the purpose of 'conversation' but to be added to the reference list in someone else's publishing activity.

However, these are the conditions of scientific conversation today. As indicated by the etymology the very concept of journal (and by the synonymous expression Zeitschrift), a journal has to be "á jour" with the contemporary conditions of a public conversation in its field. We find that an open access journal fits quite well into these conditions. EJWI's ambition is to contribute to, and stimulate, a larger conversation on workplace-related issues of innovation. The articles to be published in this journal therefore will address a larger audience than those whose research efforts are dedicated to pursue just a particular approach, 'strand' or 'school' within innovation and workplace-oriented research. The principle of 'open access' means that EJWI is most easily accessed by those who will take an interest in participating in this conversation. Thus, the most important task for the editors is to make sure that the articles we publish really are interesting to those who would like to participate.

\section{Reference}

Fagerberg J. and Verspagen B. (2009): Innovation studies - The emerging structure of a new scientific field. Research Policy 38: 218-233 
